\title{
Estudo eletrocardiográfico de equinos de salto sadios ${ }^{1}$
}

\author{
Mariana P. Diniz ${ }^{2}$, Lilian E.S. Michima ${ }^{3}$ e Wilson R. Fernandes ${ }^{4}$
}

\begin{abstract}
Diniz M.P., Michima L.E.S. \& Fernandes W.R. 2011. [Electrocardiographic study in healthy showjumping horses.] Estudo eletrocardiográfico de equinos de salto sadios. Pesquisa Veterinária Brasileira 31(4):355-361. Departamento de Clínica Médica, Faculdade de Medicina Veterinária e Zootecnia, Universidade de São Paulo, Av. Prof. Dr. Orlando Marques de Paiva 87, Bloco 12/14, São Paulo, SP 05508-270, Brazil. E-mail: marianaperesdiniz@yahoo.com.br

The electrocardiographic study was made in 100 healthy showjumping horses, with ages between 4 and 19 year-old, being 61 males and 39 females, with mean bodyweight of $516.3 \mathrm{~kg}$. A mean heart rate of $40.20 \pm 13.33 \mathrm{bpm}$ was observed, and the most common cardiac rhythm was sinus rhythm (56\%). Alterations in cardiac rhythm were observed in $38 \%$ of the animals, as wandering pacemaker (22\%), second degree atrioventricular block $(4 \%)$, first degree atrioventricular block (3\%), ventricular premature contraction ( $2 \%)$, atrial premature contraction (1\%). There was no relationship between the variables (heart rate, rhythm and arrhythmias) and the athletic performance, age and sex. The heart score data obtained after statistical analysis were not different concerning the athletic performance, age or sex. Therefore, heart score, in this sample, did not appear to be a confident parameter to predict the athletic future or the training level of the individual horse.
\end{abstract}

INDEX TERMS: Electrocardiography, heart score, horses

RESUMO.- Avaliou-se o eletrocardiograma de 100 equinos sadios praticantes de hipismo clássico modalidade salto, com idades entre 4 e 19 anos, sendo 61 machos e 39 fêmeas, com média de $516,3 \mathrm{~kg}$. Observou-se frequência cardíaca média de 40,20 $\pm 13,33 \mathrm{bpm}$, sendo o ritmo cardíaco mais freqüente o sinusal (56\%). As alterações de ritmo cardíaco estiveram presentes em $38 \%$ dos animais estudados, sendo a mais presente o marcapasso migratório (22\%), seguido de bloqueio atrioventricular de $2^{\circ}$ grau (4\%), blo-

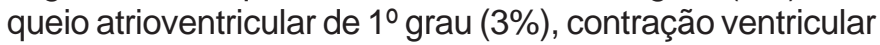
prematura $(2 \%)$, contração atrial prematura (1\%). Não se observou correlação entre as variáveis estudadas (freqüên-

${ }^{1}$ Recebido em 5 de outubro de 2010.

Aceito para publicação em 24 de janeiro de 2011.

Este artigo é parte da Dissertação de Mestrado da primeira autora, realizado no Departamento de Clínica Médica, Faculdade de Medicina Veterinária e Zootecnia, Universidade de São Paulo (USP), Brasil.

2 Médica Veterinária, Rua Álvaro Moreira 355, Barra da Tijuca, Rio de Janeiro, RJ 22630-160, Brasil. *Autor para correspondência: marianaperesdiniz@yahoo.com.br

${ }^{3}$ Médica Veterinária, Avenida Prof. Castro Jr 491, Vila Sabrina, São Paulo, SP, 02138-030.

${ }^{4}$ Departamento de Clínica Médica, Faculdade de Medicina Veterinária e Zootecnia, Universidade de São Paulo (USP), Av. Prof. Dr. Orlando Marques de Paiva 87, Bloco 12/14, São Paulo, SP 05508-270. cia cardíaca, ritmo e arritmias) e a capacidade atlética, a idade e o sexo. O escore cardíaco após analise estatística não diferiu entre os grupos quanto ao desempenho atlético, a faixa etária e o sexo. O escore cardíaco, nesta amostra, não se mostrou um parâmetro confiável para se predizer o futuro atlético ou o nível de treinamento do eqüino.

TERMOS DE INDEXAÇÃO: Eletrocardiografia, escore cardíaco, equinos.

\section{INTRODUÇÃO}

A cardiologia veterinária vem assumindo grande importância no universo da medicina esportiva principalmente em eqüinos, não só pela detecção das alterações cardíacas e tratamentos adequados, mas também por estabelecer prognósticos tanto na vida atlética como na sobrevida, racionalizando a campanha atlética ou a atividade a que se destina o animal. Porém, o uso da eletrocardiografia ainda desperta dúvidas, pois a orientação, amplitude e duração das ondas do ECG dependem de vários fatores, incluindo idade do animal, raça, sexo, derivação examinada e até mesmo condicionamento físico, e ainda, a falta de dados específicos para cada raça ou esporte (Reef 1992).

O eletrocardiograma é o método diagnóstico mais adequado para a confirmação dessas arritmias (Belgrave 1990). 
Este é um método diagnóstico que pode ser utilizado com relativa facilidade a campo, de resultado imediato, com baixo custo e que oferece informações valiosas sobre a atividade elétrica cardíaca. Entretanto, deve estar inserido num exame clínico minucioso, podendo ser indicado em situações de suspeita de arritmia durante exame clínico, mas também de forma preventiva em exames de compra, risco cirúrgico, avaliação de condicionamento físico e racionalização de campanha atlética. O eletrocardiograma na espécie eqüina é menos preciso na avaliação de tamanho das câmaras cardíacas em virtude de algumas particularidades da despolarização cardíaca dessa espécie (Reed \& Bayly 2000).

Para realização do exame é necessário que se conheça o padrão eletrocardiográfico, porém, os padrões atuais da eletrocardiografia em equinos nem sempre são adequados aos animais pesquisados, devido aos fatores que podem influenciar os traçados. Esse padrão é determinado em animais normalmente atletas, mas não considera o tipo de atividade física exercida pelo eqüino e nem as condições ambientais e de alimentação a que este é submetido.

Segundo Robertson (1992), em comparação com outras espécies domésticas, os equinos saudáveis têm uma alta incidência de arritmias cardíacas. Várias dessas são fisiológicas, porém outras podem vir acompanhadas de baixo desempenho atlético (Mitten 1996). Algumas arritmias podem ser reconhecidas pela auscultação, mas só podem ser realmente confirmadas pelo exame eletrocardiográfico (Belgrave 1990).

As arritmias consideradas fisiológicas, podendo ser abolidas com o exercício, incluem: bloqueios atrioventriculares (BAV) de $1^{\circ}$ e $2^{\circ}$ graus, marcapasso migratório e bradicardia sinusal e as arritmias que podem levar à queda de performance física são os complexos atriais e ventriculares prematuros, BAV de $2^{\circ}$ grau avançado, BAV de $3^{\circ}$ grau e fibrilação atrial.

Os problemas cardiovasculares, segundo Reef (2001), são a terceira causa mais comum de intolerância ao exercício em equinos atletas de elite, atrás apenas dos problemas músculo-esqueléticos e respiratórios. As alterações cardiovasculares são geralmente parte de um processo multifatorial, mas podem vir como única causa da queda de performance. Vários destes problemas são subclínicos, sendo portanto pouco diagnosticados na prática clínica. Dentre as causas cardiovasculares, as arritmias cardíacas são de grande importância, principalmente em animais atletas.

Particularmente, a modalidade salto do hipismo clássico, por mesclar salto e corrida, gera necessidade de condicionamento físico adequado por parte dos equinos além de grande demanda do sistema cardiovascular, podendo portanto propiciar o desenvolvimento dessas arritmias. Porém, não existem dados específicos para esta modalidade esportiva (salto). Assim, sentiu-se a necessidade do estudo eletrocardiográfico em equinos clinicamente sadios, para avaliação do comportamento eletrocardiográfico dessa população e estabelecimento de padrões de normalidade, além de estudar a possível relação da intensidade do exercício e/ ou da idade do animal como causas de alterações de ritmo cardíaco.

\section{MATERIAL E MÉTODOS}

Foram utilizados 100 equinos de hipismo clássico, praticantes da modalidade salto, representados por 61 machos e 39 fêmeas, com idades entre quatro e 19 anos e peso médio de 516,3 $\mathrm{kg}$. Todos os animais eram sadios, sem qualquer queixa de queda de desempenho atlético por parte de seus proprietários.

O exame eletrocardiográfico foi realizado após exame físico, segundo técnica descrita por Fregin (1985), utilizando um aparelho de eletrocardiografia portátil FUNBEC (ECG-4), composto por seis canais, três sensibilidades e velocidades de $25 \mathrm{~mm} / \mathrm{s}$ e $50 \mathrm{~mm} / \mathrm{s}$. Os registros foram feitos com os animais em posição ortostática, em velocidade $25 \mathrm{~mm} / \mathrm{s}$ e sensibilidade de $1 \mathrm{~cm}=1 \mathrm{mV}$. Foram registradas as derivações bipolares I, II e III e as derivações unipolares amplificadas (aVR, aVF, aVL).

Os eletrodos foram conectados à pele do animal por meio de condutores metálicos (clipes tipo jacaré), após a colocação de um algodão embebido em álcool. Foram feitas as mensurações em todas as derivações, mas como critério de avaliação e comparação, utilizou-se a derivação II como base.

Para avaliar a influência relacionada à capacidade atlética (intensidade do exercício), os equinos foram agrupados em duas classes, sendo um grupo composto por 56 animais, que saltam até $1,20 \mathrm{~m}$, e outro grupo composto por 44 animais, que saltam acima de $1,20 \mathrm{~m}$. Com relação à influência etária, os animais foram divididos em três classes, de 4-8 anos, 9-13 anos e 14-19 anos.

Calculou-se o escore cardíaco por meio da média aritmética do complexo QRS nas derivações I, II e III, segundo descrições de Steel (1963). Calculou-se média, desvio-padrão, valor mínimo e máximo para as variáveis numéricas. Realizou-se o teste ANOVA (ou t para amostras independentes) para comparação entre grupos, complementado pelo teste Tukey, para a avaliação das influências relacionadas aos fatores de capacidade atlética, sexuais e etários. Utilizou-se o teste do qui-quadrado para a avaliação das variáveis qualitativas, sempre considerando-se o nível de significância de 5\% (pd"0,05). Os cálculos foram feitos por meio do programa estatístico Epi info 5 .

\section{RESULTADOS E DISCUSSÃO}

A frequência cardíaca média encontrada foi de 40,20 $\pm 13,33$ bpm, confirmando estudos de Fregin (1992), que referem a FC entre 26-50 bpm, e de Hilwig (1977), cujos resultados foram de $22-50 \mathrm{bpm}$. O valor mínimo encontrado foi de 18,50 bpm e o valor máximo, 89,45 bpm.

A freqüência cardíaca é um parâmetro que pode sofrer variações quanto ao esporte, sexo e raça animal. Este parâmetro apresentou-se mais elevado em equinos de salto, do que em estudos realizados por Michima (2003) e Fernandes (1994), com cavalos de enduro da raça Árabe e por Fregin (1982), em equinos de corrida.

Não houve diferença entre os grupos segundo a atividade física $(p=0,1772)$, sexo $(p=0,4423)$ ou idade $(p=0,7563)$, semelhante aos resultados de Vincenzi (1995) que trabaIhou com equinos Mangalarga e não observou diferenças quanto ao sexo. Contudo, difere dos resultados obtidos por Fernandes et al. (2004), em que a freqüência cardíaca dimi-

\footnotetext{
${ }^{5}$ Epi Info Software, versão 3.4.3, 2007.
} 


\section{Quadro 1. Alterações do ritmo cardíaco encontradas no eletrocardiograma de equinos de salto}

\begin{tabular}{|c|c|}
\hline Alteração do ritmo cardíaco & $\begin{array}{l}\text { Porcentagem } \\
(\%)\end{array}$ \\
\hline Marcapasso migratório & 22,0 \\
\hline Bloqueio atrioventricular de $1^{\circ}$ grau & 3,0 \\
\hline Bloqueio atrioventricular de $2^{\circ}$ grau & 4,0 \\
\hline Bloqueio atrioventricular de $1^{\circ}$ e $2^{\circ}$ grau & 3,0 \\
\hline $\begin{array}{l}\text { Marcapasso migratório e bloqueio atrioventricular } \\
\text { de } 2^{\circ} \text { grau }\end{array}$ & 1,0 \\
\hline $\begin{array}{l}\text { Marcapasso migratório e bloqueio atrioventricular } \\
\text { de } 1^{\circ} \text { e } 2^{\circ} \text { grau }\end{array}$ & 1,0 \\
\hline Marcapasso migratório e "sinus arrest" & 1,0 \\
\hline Contração atrial prematura & 1,0 \\
\hline Contração ventricular prematura & 2,0 \\
\hline Sem alterações & 62,0 \\
\hline Total & 100,0 \\
\hline
\end{tabular}

nuiu com a idade, havendo diferença entre potros até um ano e os animais de sobreano. Como no presente trabalho os animais tinham acima de quatro anos, ou seja, adultos, talvez seja essa a explicação para não haver diferença entre os grupos segundo faixa etária nessa variável, já que os equinos em questão já atingiram quase que o máximo de desenvolvimento corpóreo e cardiovascular.

O ritmo cardíaco mais freqüente foi o sinusal (56\%), seguido da taquicardia sinusal (23\%), arritmia sinusal (20\%) e bradicardia sinusal (1\%), resultados que se assemelham aos de Fernandes et al. (2004), que encontraram nos animais sobreanos o ritmo sinusal como mais freqüente, seguido de taquicardia sinusal e arritmia sinusal, diferentemente dos resultados de Ayala et al. (1995) em equinos da raça Andaluz, nos quais o ritmo mais freqüente foi a arritmia sinusal, seguida do ritmo sinusal. Não houve diferença entre os grupos segundo atividade física $(p=0,4621)$, sexo $(p=0,4833)$ ou idade $(p=0,5800)$.
A arritmia sinusal pode ser normalmente encontrada nos animais em repouso, podendo ter relação com o ciclo respiratório e assim como a bradicardia sinusal em equinos, pode também estar associada ao alto tônus vagal. Já a taquicardia sinusal, na ausência de outros sinais clínicos, pode estar associada a excitação do animal, o que pode ocorrer durante a realização do exame (Fregin 1992).

Quanto às alterações de ritmo encontradas (Quadro 1), observou-se que $38 \%$ dos animais apresentaram pelo menos um tipo de alteração do ritmo cardíaco. Também não foi observada diferença significativa entre os grupos segundo atividade física $(p=0,3300)$, sexo $(p=0,4931)$ ou idade $(p=0,7572)$ em relação às alterações de ritmo cardíaco.

A maioria dos autores consultados concorda que o marcapasso migratório (Fig.1), os bloqueios atrioventriculares de $1^{\circ}$ e $2^{\circ}$ graus (Fig.2) e o bloqueio sinoatrial/"sinus arrest" (Fig.3) são arritmias fisiológicas em equinos, sendo algumas influenciadas pelo alto tônus vagal, desaparecendo com o exercício (Glazier 1958, Hilwig 1977, Reimer 1992, King et al. 1994, Mitten 1996). No entanto, existem autores que descrevem o BAV de $2^{\circ}$ grau como motivo de queda de performance atlética (Steel 1963).

Segundo Hilwig (1977), Fregin (1982) e Raekallio (1992), o marcapasso migratório pode estar presente em $30 \%$ dos equinos saudáveis e em repouso, enquanto o BAV de $2^{\circ}$ grau pode estar presente em 15 a $18 \%$ dos equinos.

Contrações atriais e ventriculares prematuras geralmente são indícios de doenças miocárdicas e podem, ainda, predispor ao desenvolvimento de fibrilação atrial (Reimer 1992). Porém, segundo Mitten (1996), as contrações atriais prematuras podem ocorrer em equinos em repouso, sem estar associada à intolerância ao exercício e, ainda, diminuir ou desaparecer durante o exercício. Já o complexo ventricular prematuro (Fig.4 e 5), é indicativo de doença

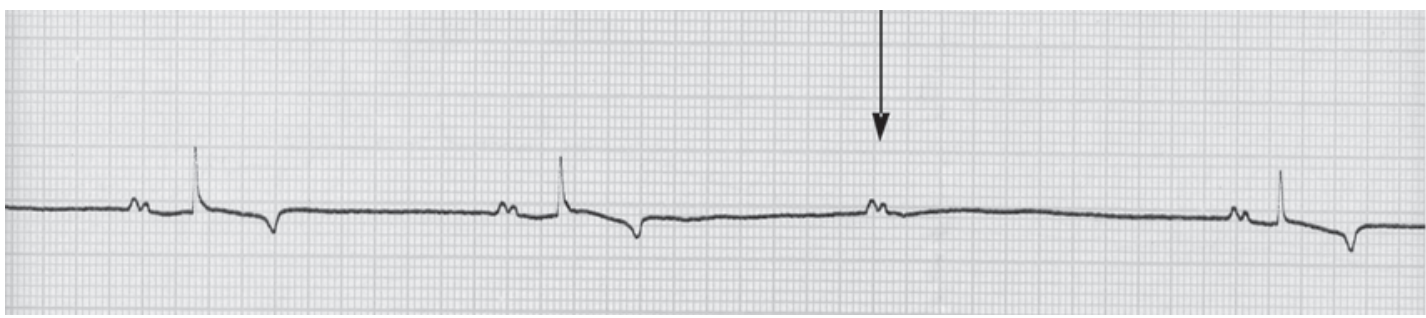

Fig.1. Eletrocardiograma de eqüino macho da raça Brasileiro de Hipismo com 10 anos, com marcapasso migratório na derivação bipolar II. Note-se a diferença de conformação das ondas P (setas). Velocidade: $25 \mathrm{~mm} / \mathrm{s}$. Sensibilidade: $\mathrm{cm}=1 \mathrm{mV}$.

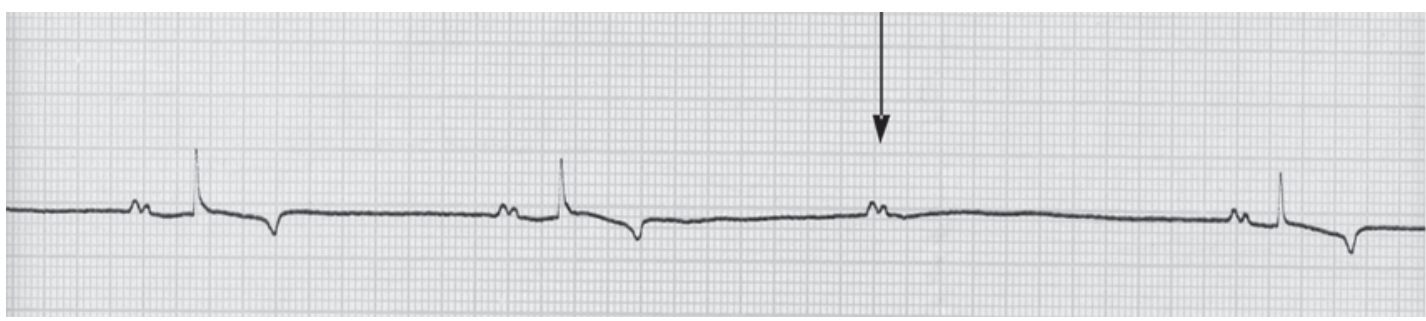

Fig.2. Eletrocardiograma de eqüino fêmea da raça Brasileiro de Hipismo com 9 anos, com bloqueio atrioventricular de $2^{\circ}$ grau na derivação unipolar aumentada aVL. Note-se a presença de uma onda P desacompanhada de complexo QRS. Velocidade: $25 \mathrm{~mm} / \mathrm{s}$. Sensibilidade: $1 \mathrm{~cm}=1 \mathrm{mV}$. 


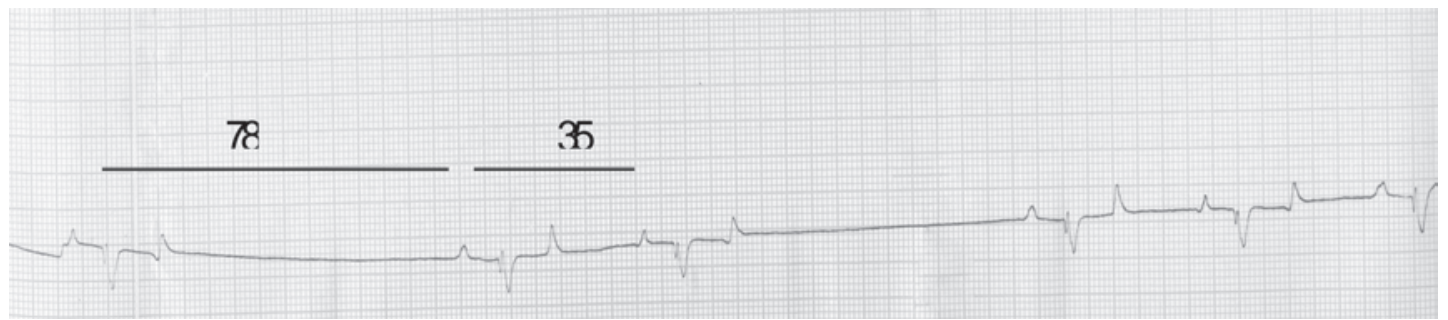

Fig.3. Eletrocardiograma de eqüino fêmea da raça Anglo-Árabe com 5 anos, com bloqueio sinoatrial/ "sinus arrest" na derivação bipolar I. Note-se que o intervalo R-R do primeiro complexo (primeiro traço) é mais que o dobro do segundo complexo (segundo traço). Velocidade: $25 \mathrm{~mm} / \mathrm{s}$. Sensibilidade: $1 \mathrm{~cm}=1 \mathrm{mV}$.

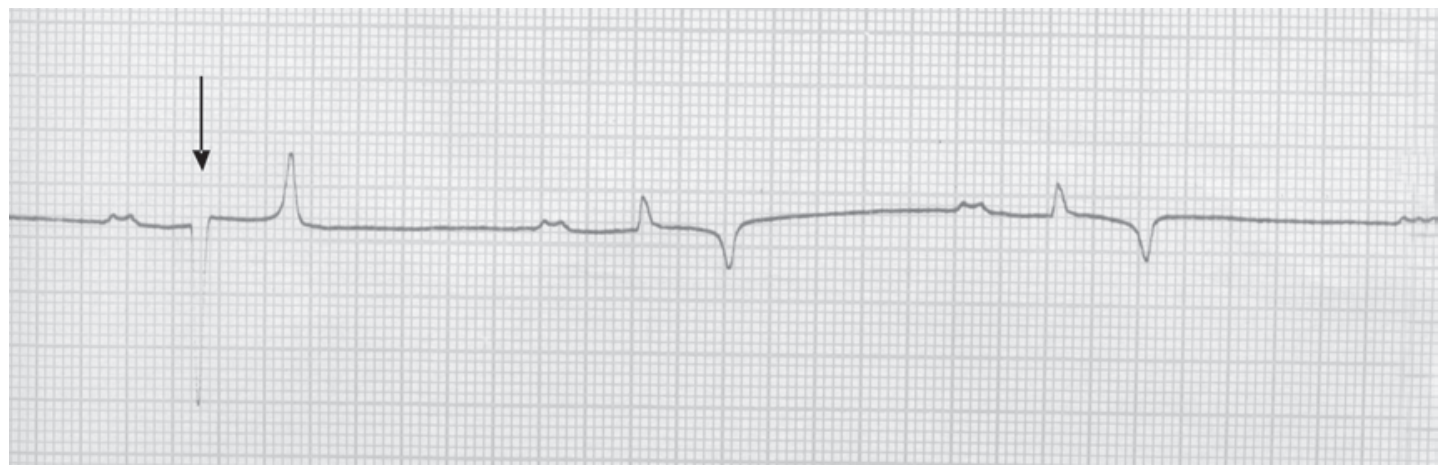

Fig.4. Eletrocardiograma de eqüino macho da raça Hanoverano com 10 anos, com contração ventricular prematura na derivação bipolar II. Note-se a presença de complexo QRS de conformaç̧ão bizarra (seta). Velocidade: $25 \mathrm{~mm} / \mathrm{s}$. Sensibilidade: $1 \mathrm{~cm}=1 \mathrm{mV}$.

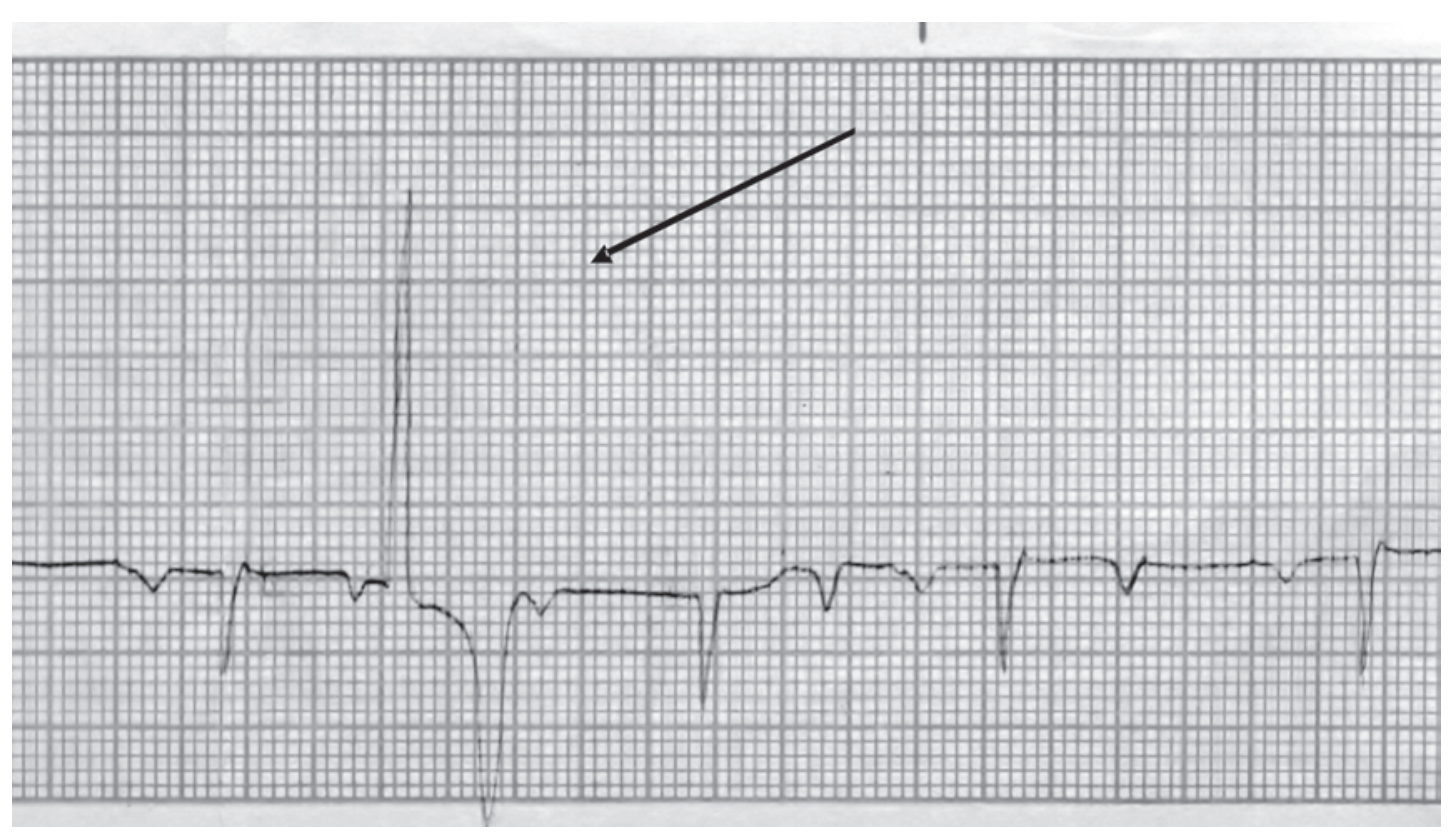

Fig.5. Eletrocardiograma de eqüino fêmea da raça Zangerchaid com 8 anos, com contração ventricular prematura na derivação unipolar aumentada aVF. Note-se a presença de complexo QRS precoce, largo, de conformação bizarra (seta). Velocidade: $25 \mathrm{~mm} / \mathrm{s}$. Sensibilidade: $1 \mathrm{~cm}=1 \mathrm{mV}$.

miocárdica, mesmo sem sinais clínicos de doenças cardiovasculares (Mitten 1996) e segundo Ryan et al. (2005), as contrações ventriculares e supraventriculares prematuras podem ser induzidas pelo exercício, em animais saudáveis. No presente trabalho, os equinos que apresenta- ram essas alterações não tinham qualquer queixa de queda de desempenho atlético por parte de seus proprietários, mas isso não descarta a possibilidade de problemas cardíacos, já que não foram feitos outros exames como ecocardiograma e eletrocardiograma pós exercício e, tal- 
Quadro 2. Frequência relativa (em porcentagem), valores médios, desvio-padrão, valor mínimo e valor máximo (em segundos) da duração das ondas e intervalos na derivação bipolar II. Obtenção pelo registro eletrocardiográfico de equinos de salto

\begin{tabular}{lccccc}
\hline Parâmetro & $\begin{array}{c}\text { Freqüência } \\
\text { relativa(\%) }\end{array}$ & Média(s) & $\begin{array}{c}\text { Desvio } \\
\text { padrão(s) }\end{array}$ & $\begin{array}{c}\text { Mínimo } \\
(\mathrm{s})\end{array}$ & $\begin{array}{c}\text { Máximo } \\
(\mathrm{s})\end{array}$ \\
\hline Onda P & 100 & 0,122 & 0,026 & 0,040 & 0,160 \\
Intervalo P-R & 100 & 0,314 & 0,074 & 0,160 & 0,520 \\
Complexo QRS & 100 & 0,091 & 0,025 & 0,040 & 0,160 \\
Onda Q & 65 & 0,026 & 0,011 & 0,020 & 0,080 \\
Onda R & 100 & 0,058 & 0,023 & 0,020 & 0,120 \\
Onda S & 41 & 0,036 & 0,014 & 0,020 & 0,080 \\
Intervalo QT & 100 & 0,491 & 0,054 & 0,320 & 0,600 \\
Onda T & 100 & 0,113 & 0,033 & 0,040 & 0,200
\end{tabular}

vez esses animais pudessem apresentar melhor performance se não tivessem estas alterações de ritmo.

A utilização do ECG nos equinos atletas mostrou-se de grande valia, tendo em vista que aproximadamente $3 \%$ dos animais examinados apresentaram arritmias que são consideradas indícios de doenças miocárdicas, sem qualquer outro sintoma, o que torna importante a realização periódica de exames cardiovasculares, incluindo exames complementares, como o eletrocardiograma, em equinos de esporte.

Os dados de duração das ondas, intervalos e complexos encontram-se no Quadro 2 e assemelham-se aos observados por Glazier (1958), porém diferem dos obtidos por Fregin (1982), em cavalos de corrida, que encontrou onda P de $0,14 \mathrm{~s}$, intervalo PR de 0,33 s, complexo QRS de 0,13 s e intervalo QT de 0,51s.

Os resultados dos equinos de salto aproximam-se dos obtidos por Ayala et al. (1995), em cavalos da raça Andaluz, em que os autores encontraram os seguintes parâmetros de duração: onda P, 0,105s; intervalo PR, 0,258s; complexo QRS, 0,095s; onda T, 0,126s e intervalo QT, 0,465s. As durações médias são maiores (exceto para a duração do complexo QRS) que as observadas por Vincenzi et al. (2000), em equinos adultos da raça Mangalarga, em que a duração de P foi de 0,07 s, intervalo PR de 0,26s, complexo QRS de 0,097s, intervalo QT de 0,39s e onda T de 0,08s.

Quanto a variável idade, houve diferenças significativas na duração do complexo QRS em aVL $(p=0,0395)$, sendo maior nos animais entre 9 e 13 anos e na duração da onda R em DI ( $p=0,0290)$, sendo maior entre o grupo de 4 a 8 anos. Em relação à atividade física desempenhada, houve diferenças estatísticas quanto à duração do intervalo QT nas derivações DI, DIII e aVF, valores de $p=0,0469$, $p=0,0263$ e $p=0,0076$, respectivamente. O intervalo $Q T$ representa o período de tempo entre o início da despolarização ventricular até sua completa repolarização. Este intervalo varia inversamente à freqüência cardíaca (Edwards 1987). Esta, talvez, seja a explicação para o ocorrido, já que a média do intervalo QT no Grupo 1 foi maior que no Grupo 2 nas derivações citadas e a freqüência cardíaca média do Grupo 1 foi menor que a do Grupo 2.

A morfologia, duração e amplitude das ondas, interva- los e segmentos podem sofrer influências de acordo com idade, raça e sexo, segundo estudo realizado com 150 equinos da raça Mangalarga, no qual se observou influência dos fatores etários na duração das ondas $\mathrm{Pe} \mathrm{T}$ (Vincenzi et al. 2000).

Quanto à variável sexo, as análises estatísticas que apresentaram diferenças significativas quanto à duração, foram o intervalo $P-R$ na derivação unipolar $\operatorname{aVR}(p=0,0497)$, onda $S$ na derivação bipolar I $(p=0,0231)$, e onda T na derivação bipolar II $(p=0,0106)$, dados estes que divergem dos de Vincenzi et al. (2000), em estudo realizado com equinos da raça Mangalarga, onde não foram observadas diferenças estatísticas em relação ao sexo quanto à duração da onda $P$, do complexo QRS, da onda T e dos intervalos $P R$ e $Q T$.

Os resultados de amplitude média das diferentes ondas apresentadas pelos equinos de salto estudados encontram-se no Quadro 3. As amplitudes das ondas Q, R, $\mathrm{S}$ e $\mathrm{T}$ mostraram-se mais elevadas que às obtidas por Ayala et al. (1998) em equinos adultos da raça Andaluz, e também às de Vincenzi et al. (2000), em que a onda $P$ apresentou $0,16 \mathrm{mV}$, onda $\mathrm{Q} 0,08 \mathrm{mV}$, onda $\mathrm{R} 0,58 \mathrm{mV}$ e onda S $0,09 \mathrm{mV}$.

A diminuição da amplitude do complexo QRS, segundo White \& Rhode (1974), pode estar associada a pleurites crônicas, efusões pericárdicas, pericardites, anemia e hipoproteinemia. A depressão do segmento ST, maior que $0,3 \mathrm{mV}$ nas derivações bipolares, pode estar associada à doença abdominal aguda incluindo peritonite e obstrução intestinal com comprometimento vascular. Ela pode, ainda, estar associada a condições de anestesia e a casos de choque. A onda T quando se encontra maior que $1,4 \mathrm{mV}$ nas derivações bipolares pode estar associada à miocardite ou hiperpotassemia. Não foram observadas depressões no segmento ST nos equinos de salto com a amplitude descrita acima e ondas T com amplitudes superiores aos valores citados.

Em relação à amplitude, houve diferença estatística na onda $R$ nas derivações bipolar III $(p=0,0325)$ e unipolares aumentadas aVR e aVF ( $p=0,0074$ e $p=0,0209$, respectivamente), e, por fim, houve diferença estatística na amplitude da onda $S$ na derivação $\operatorname{aVL}(p=0,0136)$. A onda $R$ apresentou tendência ao aumento de acordo com a idade,

Quadro 3. Frequência relativa (em porcentagem), valores médios, desvio-padrão, valor mínimo e valor máximo (em milivolts), da amplitude das ondas na derivação bipolar II, obtidos pelo registro eletrocardiográfico de equinos de salto

\begin{tabular}{lccccc}
\hline Parâmetro & $\begin{array}{c}\text { Freqüência } \\
\text { relativa }(\%)\end{array}$ & $\begin{array}{c}\text { Média } \\
(\mathrm{mV})\end{array}$ & $\begin{array}{c}\text { Desvio } \\
\text { padrão }(\mathrm{mV})\end{array}$ & $\begin{array}{c}\text { Mínimo } \\
(\mathrm{mV})\end{array}$ & $\begin{array}{c}\text { Máximo } \\
(\mathrm{mV})\end{array}$ \\
\hline Onda P única & 35 & 0,267 & 0,075 & 0,100 & 0,500 \\
Onda P bifásica & 11 & 0,314 & 0,110 & 0,200 & 0,600 \\
Onda P1 & 54 & 0,135 & 0,049 & 0,100 & 0,250 \\
Onda P2 & 54 & 0,226 & 0,050 & 0,100 & 0,300 \\
Onda Q & 65 & 0,192 & 0,311 & 0,050 & 2,400 \\
Onda R & 100 & 1,022 & 0,503 & 0,100 & 2,800 \\
Onda S & 41 & 0,126 & 0,060 & 0,050 & 0,300 \\
Onda T & 100 & 0,442 & 0,204 & 0,100 & 0,400
\end{tabular}


o que não foi observado por Fernandes et al. (2004), que não encontraram diferenças estatísticas em relação às amplitudes entre os diferentes grupos etários de equinos da raça PSI.

Os grupos divididos segundo a atividade física apresentaram diferenças estatísticas na amplitude da primeira porção da onda $P(P 1)$ na derivação $\mathrm{DI}(\mathrm{p}=0,0218)$ e $\mathrm{aVL}$ $(p=0,0456)$ e na amplitude da onda T em DI $(p=0,0251)$. Não se encontraram dados comparativos na literatura, que comprovassem a existência de diferenças eletrocardiográficas relacionadas a intensidade de esforço físico dentro de uma mesma atividade esportiva.

Em relação ao fator sexual, as variáveis de amplitude que apresentaram diferenças estatísticas foram a onda $P$ única nas derivações unipolares $\mathrm{aVL}(\mathrm{p}=0,0107)$ e $\mathrm{aVF}$ $(p=0,0019)$, a segunda porção da onda $P$ bífida (P2) na derivação bipolar III ( $p=0,0007)$, a onda $Q$ na derivação bipolar I $(p=0,0078)$ e a onda T nas derivações bipolar II $(p=0,0082)$ e unipolar aVF $(p=0,0490)$. Estes dados diferem dos resultados obtidos por Ayala et al. (1998) e Vincenzi et al. (2000), em que não foram observadas diferenças estatísticas em relação ao fator sexual quanto à amplitude das ondas $P, Q, R, S$ e $T$.

O escore cardíaco, como índice de potencial de performance atlética, foi bastante estudado por Steel (1963) e Steel \& Stewart (1974) em cavalos de corrida e também por Rose et al. (1979) e Illera \& Illera (1987) em equinos de enduro. Posteriormente, Rose et al. (1980) também observaram a mesma correlação em cavalos Puro Sangue Inglês, que competiam em eventos de três dias, tendo encontrado um escore médio de 120 milissegundos, o que os levou a concluir a necessidade de se ter alto escore cardíaco para se competir com sucesso, neste tipo de prova. Porém, Leadon et al. (1991) em seu estudo com equinos PSI, não observaram relação entre o escore cardíaco e os parâmetros ecocardiográficos e a subsequente performance atlética do animal nas corridas.

O escore cardíaco médio encontrado nos equinos de salto foi de $94,9 \pm 16,1 \mathrm{~ms}$, que é muito inferior aos índices acima descritos para cavalos de evento de três dias (Rose et al. 1980); para cavalos de trote com mais de cinco anos (105ms), segundo Nielsen e Vibe-Petersen (1980) e para cavalos de enduro nos quais Illera \& Illera (1987) encontraram escore de $100 \mathrm{~ms}$ para animais com pior condicionamento físico. Deve-se considerar as diferenças entre os esportes corrida, enduro e prova de três dias em relação ao salto; as três modalidades anteriores são mais extenuantes que o salto, a velocidade atingida durante uma prova de corrida é muito maior que nas provas de salto, e no enduro, as provas geralmente são de resistência, o que também difere da modalidade salto. Talvez, este esporte não tenha a mesma demanda cardiovascular que os demais, daí os resultados serem inferiores ou até nestes animais não haja boa correlação entre o escore cardíaco e o peso cardíaco.

Não existe na literatura consultada dados sobre a utilização do escore cardíaco para avaliar dentro de uma mesma modalidade esportiva os diferentes desempenhos, as- sim, em nosso estudo avaliando equinos que saltam abaixo de 1,20m (Grupo 1) e equinos que saltam acima de 1,20m (Grupo 2), não observamos diferenças estatísticas no escore cardíaco, o que talvez poderia ser explicado pela falta de aplicabilidade desta medida ao tipo de exercício que as provas de salto promovem, ou ainda porque 0 tipo de condicionamento físico realizado pelo Grupo 1 e 2 seja basicamente o mesmo, independentemente da altura que os animais saltam nas provas.

De acordo com Steel (1963), o escore cardíaco pode variar significativamente segundo sexo, porém, no presente estudo escores cardíacos de machos e fêmeas não apresentaram diferenças estatisticamente significativas, apesar dos machos apresentarem valores um pouco maiores. Não houve diferença estatística entre os animais segundo a faixa etária, talvez porque os animais estudados estavam acima de quatro anos, faixa etária próxima da que alguns autores descrevem como tendo pouca alteração no escore cardíaco (Nielsen \& Vibe-Petersen 1980).

\section{CONCLUSÕES}

Com este trabalho foi possível obter o padrão eletrocardiográfico de cavalos praticantes de hipismo clássico, onde a freqüência cardíaca média foi de 40,20 bpm, com ritmo mais freqüente sendo o sinusal e $38 \%$ dos animais podem apresentar algum tipo de variação de ritmo cardíaco.

A capacidade atlética, o sexo e a faixa etária podem influenciar alguns parâmetros eletrocardiográficos.

O escore cardíaco médio é de $94,9 \pm 16,1 \mathrm{~ms}$ e não sofre influências de fatores como a capacidade atlética, idade ou sexo e não parece ser um bom parâmetro para avaliação de aptidão física para equinos de hipismo modalidade salto no padrão estudado.

\section{REFERÊNCIAS}

Ayala I., Montes A., Benedito J.L., Castillo C., Hernández J., Gutierrez C. \& Garcia-Partida P. 1998. Modifications of the form and amplitude of the electrocardiographic QRS complex during growth in the Spanish-Bred horse. J. Am. Vet. Med. Assoc. 45:309-317.

Ayala I., Montes A., Bernal L.J. \& Sandoval J.A. 1995. Electrocardiographic values in Spanish-bred horses in different ages. Aust. Vet. J. 72:225-226.

Belgrave J.O.S. 1990. A case of atrial fibrillation with congestive heart failure. Equine Vet. Educ. 2:2-4.

Reed S.M. \& Bayly W.M. 2000. Doenças cardiovasculares, p.250319. In: Bonagura J.D. \& Reef V.B. (Eds), Medicina Interna Equina. Guanabara Koogan, Rio de Janeiro.

Edwards N.J. 1987. Bolton's Handbook of Canine and Feline Electrocardiography. $2^{\text {nd }}$ ed. W.B. Saunders, Philadelphia. 381p.

Epi Info Software, versão 3.4.3, 2007

Fernandes W.R. 1994. Alterações dos parâmetros do eletrocardiograma e da crase sangüínea em eqüinos das raças Árabe e Mangalarga, bem como de Mestiços, submetidos à prova de enduro. Tese de Doutorado em Medicina Veterinária, Faculdade de Medicina Veterinária e Zootecnia, Universidade de São Paulo, SP. 71p.

Fernandes W.R., Larsson M.H.M.A., Alves A.L.G., Fantoni D.T. \& Belli C.B. 2004. Características eletrocardiográficas em eqüinos clinicamente normais da raça Puro Sangue Inglês. Arq. Bras. Med. Vet. Zootec. 56:143-149. 
Fregin G.F. 1982. The equine electrocardiogram with standardized body and limb positions. Cornell Vet. 72:304-324.

Fregin G.F. 1985. Electrocardiography. Vet. Clin. North Am., Equine Pract. 1:419-435.

Fregin G.F. 1992. Medical evaluation of the cardiovascular system. Vet. Clin. North Am., Equine Pract. 8:329-346.

Glazier D.B. 1958. Electrocardiography in Veterinary Medicine. Irish Vet. J.12:230-252.

Hilwig R.W. 1977. Cardiac arrhythmias in the horse. J. Am. Vet. Med. Assoc. 170:153-163.

Illera J.C. \& Illera M.1987. Electrocardiography and heart score of horses competing in an endurance ride. Aust. Vet. J. 64: 88-89.

King C.M., Evans D.L. \& Rose R.J. 1994. Significance for exercise capacity of some electrocardiographic findings in racehorses. Aust. Vet. J. 71:200-202.

Leadon D., Mcallister H., Mullins E. \& Osborne M. 1991. Electrocardiographic and echocardiographic measurements and their relationships in Thoroughbred yearlings to subsequent performance, p.22. In: Persson S.G.B. (Ed.), Equine Exercise Physiology. $3^{\text {rd }}$ ed. ICEEP Publications, Davis.

Michima L.E.S. 2003. Avaliação de dimensões e índices cardíacos obtidos por ecocardiografia de eqüinos de enduro criados no estado de São Paulo. Dissertação de Mestrado em Medicina Veterinária, Faculdade de Medicina Veterinária e Zootecnia, Universidade de São Paulo, SP. 71p.

Mitten L.A. 1996. Cardiovascular causes of exercise intolerance. Vet. Clin. North Am., Equine Pract. 12:473-494.

Nielsen K. \& Vibe-Petersen G.1980. Relationship between QRS-duration (heart score) and racing performance in trotters. Equine Vet. J. 12:8184

Raekallio M.1992. Long term ECG recording with holter monitoring in clinically healthy horses. Acta Vet. Scand. 33:71-75.

Reef V.B. 1992. Cardiovascular problems associated with poor performance, p.381-410. In: Robinson N.E. (Ed.), Current Therapy in Equine Medicine $3^{\text {rd }}$ ed. W.B. Saunders, London.
Reef V.B. 2001. Stress echocardiography and its role in performance assessment. Vet. Clin. North Am., Equine Pract. 17:179-189.

Reimer J.M. 1992. Cardiac arrhythmias, p.382-393. In: Robinson N.E. (Ed.), Current Therapy in Equine Medicine. $3^{\text {rd }}$ ed. W.B. Saunders, London.

Robertson S.A. 1992. Electrocardiography for the equine practioner. Vet. Annual 32:192-200.

Rose R.J., Backhouse J.W. \& Ilkiw J.E. 1980. Electrocardiography and haematology of horses competing in a three-day event. Aust. Vet. J. 56:318 -320

Rose R.J., Ilkiw J.E. \& Hodgdon D. 1979. Electrocardiography, heart score and haematology of horses competing in an endurance ride. Aust. Vet. J. 55:247-250.

Ryan N., Marr C.M. \& McGladdery A.J. 2005. Survey of cardiac arrhythmias during submaximal and maximal exercise in Thoroughbred racehorses. Equine Vet. J. 37:265-268.

Steel J.D. 1963. Studies on the electrocardiogram of the racehorse. Australian Medical Publishing Co., Sydney. 48p.

Steel J.D. \& Stewart G.A. 1974. Electrocardiography of the horse and potential performance ability. J. South Afr. Vet. Assoc. 45:263268.

Stewart G.A. 1981. The heart score theory in the racehorse. Aust. Vet. J. 57:422-428.

Vincenzi R.C. 1995. Determinação dos parâmetros eletrocardiográficos de eqüinos da raça Mangalarga, criados no Estado de São Paulo. Dissertação de Mestrado em Medicina Veterinária, Faculdade de Medicina Veterinária e Zootecnia, Universidade de São Paulo, SP. 73p.

Vincenzi R.C., Larsson M.H.M.A. \& Fernandes W.R. 2000. Parâmetros eletrocardiográficos em eqüinos clinicamente normais da raça Mangalarga. III. Amplitude e duração dos complexos e intervalos. Revta Bras. Med. Vet. 22:194-198.

White II N.A. \& Rhode E.A. 1974. Correlation of electrocardiographic findings to clinical disease in the horse. J. Am. Vet. Med. Assoc. 164:46-56. 